\title{
An Integration Scheme of the Primitive Equation Model with an Icosahedral-Hexagonal Grid System and its Application to the Shallow Water Equations
}

\author{
By Yoshinobu Masuda* and Haruo Ohnishi \\ Meteorological Research Institute, Tsukuba, Ibaraki 305, Japan \\ (Manuscript received 16 October 1986, in revised form 12 February 1987)
}

\begin{abstract}
A numerical scheme for integrating the primitive equation over the spherical earth with a quasi-uniform grid system, which is an icosahedral-hexagonal grid, is presented. Making use of the stream function and the velocity potential, all the components of the primitive equations are expressed by Jacobian, Laplacian and flux divergence terms. The finite difference forms for these terms are represented by the line-integral which is easy to form a conserving scheme. The scheme presented here conserves exactly the total mass and almost exactly the total energy and absolute potential enstrophy of a simple divergent flow. 100 days forecast was performed by this grid system with the Rossby-Haurwitz wave as the initial condition. The total mass was conserved satisfactorily, and the conservation of total energy was also achieved within $0.001 \%$ error throughout 100 days integration. The error in the conservation of total absolute potential enstrophy is $0.01-0.1 \%$, and the results of 100 days forecast are satisfactory.
\end{abstract}

\section{Introduction}

For numerical integration of the equations of atmospheric motion in the spherical domain, it is desirable to use a quasi-uniform grid which divides the sphere into elements that are nearly equal in area and in shape. That is, basically, the grid should be as uniform as possible and should preserve the isotropy of the sphere around any grid point as well as possible.

Gates and Riegel (1962) and Kurihara (1965) have proposed the global grid systems which have less grid points around the latitude circle at higher latitudes and maintain quasi-uniform grid size all over the sphere. Arakawa (1966) emphasized that the integral constraints which hold for the differential form of the original equation should also be maintained in the

\footnotetext{
* Present address : Izumi-hon-cho, 2-18-13, Komae, Tokyo 201

(Retired from Meteorological Research Institute)
}

finite difference analogue of this equation. The integral constraints which are maintained in his scheme are the kinetic energy, total vorticity and total square vorticity in the barotropic case. When these properties are conserved, nonlinear computational instability is prevented. Moreover, the spectral distribution of the kinetic energy is constrained to maintain its average scale. The schemes proposed by Gates et al. and Kurihara, which did not obey these properties, are no longer used.

Sadourny et al. (1968) and Williamson (1968) indepedently proposed the schemes for integration of the barotropic vorticity equation on a spherical geodesic grid. In their schemes the sphere is divided into 20 major spherical triangles to form a spherical icosahedron and each triangular face further subdivided into smaller triangles (Fig. 1). They have got a finite difference form of the Jacobian or advection term of the barotropic equation which 


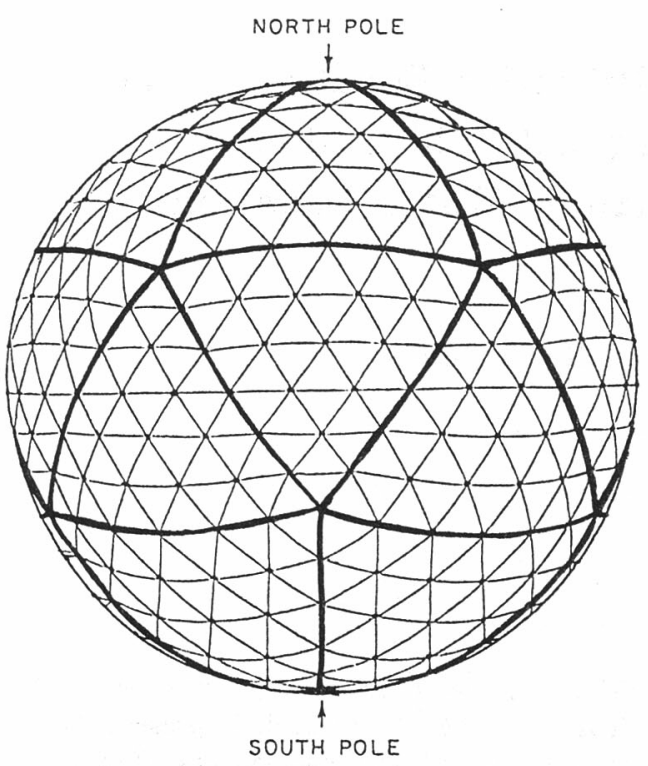

Fig. 1 Representation of the icosahedral-hexagonal grid for 6 subdivisions. From Sadourny et al. (1968)

conserves the total kinetic energy, total vorticity and total square vorticity. The scheme was tested in the flows of known analytical solutions. They obtained more favorable results than those of the more usual spherical grid in those days.

Since then the icosahedral-hexagonal grid was extended to the integration of the primitive equations over the globe. For example many efforts were made by Sadourny and Morel (1969), Masuda (1969), Sadourny (1969) and Williamson (1969) individually. They tried to apply the governing equations of momentum form to the hexagonal grid directly and to develop a conservative finite-difference scheme. It was difficult, however, to derive a numerical scheme conserving total potential enstrophy, which corresponds to total square vorticity in the non-divergent case. And then, all of them failed in the long-term numerical integrations with their hexagonal grids. As a result, the geodesic grid system has been given up for a long time. Only Sadourny (1975), apart from the hexagonal grid, succeeded in the global numerical experiments by using the potential enstrophy conserving scheme on the cylindrical coordinates. This approach has the disadvantage that a large discrepancy between longitudinal and latitudinal grid spacing arises in the vicinity of the poles, which means greater anisotropy for the calculations at higher latitudes.

In recent years, the spectral method has been widely used for the numerical integration of the global primitive equation model. This method has many advantages. Theoretically it does not induce any delay of wave propagation. And also, nonlinear normal mode initialization can be easily adapted to this method. In the spectral method, however, the treatment of physical processes such as the effects of cumulus convection or airsea interaction is not so simple. Before the estimation of the effects mentioned above, the spectral expression of physical quantities must be transformed to grid values. Moreover, because any point source such as an isolated mountain is represented by a combination of many waves, unexpected errors may be generated in the regions far from the source if the spectral resolution is not large enough. Besides these problems, there is another problem in the spectral model, that the resolution near the poles becomes much smaller like in latitude-longitude grid models. It is doubtful whether the sub-grid parameterization schemes developed on a comparatively large grid size system can be applied to such a very small grid size without any modification.

For all these reasons, the icosahedralhexagonal grid model, which has nearly homogeneous grid distances, is investigated again in this study. However, to overcome the failures encountered by many authors about 20 years ago, a different way is examined. Developing the idea which was applied on the non-divergent barotropic equation by Sadourny et al. (1968) and Williamson (1968), we use the stream function and velocity potential as the dependent variables of the primitive equations instead of the velocity components. With use of these variables all the terms of the governing equations are written by Jacobian; Laplacian and flux divergence terms. So, it is easy to derive the scheme which maintains the conservation properties of original equations. The scheme 
proposed in this paper conserves exactly the total mass and total absolute potential vorticity and almost exactly the total energy and absolute potential enstrophy of a simple divergent flow.

\section{The governing equations written by the stream function and the velocity potential}

The governing equations in the $\sigma$-coordinate system on the sphere are written by

$$
\begin{aligned}
& \frac{\partial u}{\partial t}+\frac{u}{a \cos \varphi} \frac{\partial u}{\partial \lambda}+\frac{v}{a} \frac{\partial u}{\partial \varphi}+\dot{\sigma} \frac{\partial u}{\partial \sigma} \\
& -\left(f+\frac{\tan \varphi}{a} u\right) v+\frac{1}{a \cos \varphi}\left(\frac{\partial \phi}{\partial \lambda}+\sigma \alpha \frac{\partial \pi}{\partial \lambda}\right) \\
& =0, \\
& \frac{\partial v}{\partial t}+\frac{u}{a \cos \varphi} \frac{\partial v}{\partial \lambda}+\frac{v}{a} \frac{\partial v}{\partial \varphi}+\dot{\sigma} \frac{\partial v}{\partial \sigma} \\
& +\left(f+\frac{\tan \varphi}{a} u\right) u+\frac{1}{a}\left(\frac{\partial \phi}{\partial \varphi}+\sigma \alpha \frac{\partial \pi}{\partial \varphi}\right)=0 \\
& \frac{\partial \pi}{\partial t}+\frac{1}{a \cos \varphi} \frac{\partial}{\partial \lambda}(\pi u)+\frac{1}{a \cos \varphi} \frac{\partial}{\partial \varphi}(\pi v \cos \varphi) \\
& +\frac{\partial}{\partial \sigma}(\pi \dot{\sigma})=0, \\
& \frac{\partial T}{\partial t}+\frac{u}{a \cos \varphi} \frac{\partial T}{\partial \lambda}+\frac{v}{a} \frac{\partial T}{\partial \varphi}+p^{\kappa} \dot{\sigma} \frac{\partial \theta}{\partial \sigma} \\
& \quad-\frac{\sigma \alpha}{C_{p}}\left(\frac{\partial \pi}{\partial t}+\frac{u}{a \cos \varphi} \frac{\partial \pi}{\partial \lambda}+\frac{v}{a} \frac{\partial \pi}{\partial \varphi}\right)-\frac{\dot{Q}}{C_{p}} \\
& =0, \\
& \frac{\partial q}{\partial t}+\frac{u}{a \cos \varphi} \frac{\partial q}{\partial \lambda}+\frac{v}{a} \frac{\partial q}{\partial \varphi}+\dot{\sigma} \frac{\partial q}{\partial \sigma}-E=0, \\
& \frac{\partial \phi}{\partial \sigma}=-\frac{R T}{\sigma} \cdot
\end{aligned}
$$

Here, $u$ and $v$ are the longitudinal and latitudinal velocity components respectively, $\theta=$ $T / p^{\alpha}$ and all other variables are used with the ordinary meanings.

If we introduce the stream function $\psi$ and the velocity potential $\chi, u$ and $v$ are expressed by

$$
u=-\frac{1}{a} \frac{\partial \psi}{\partial \varphi}+\frac{1}{a \cos \varphi} \frac{\partial \chi}{\partial \lambda},
$$

$$
v=\frac{1}{a \cos \varphi} \frac{\partial \psi}{\partial \lambda}+\frac{1}{a} \frac{\partial \chi}{\partial \varphi} .
$$

Making use of the following operators on the spherical coordinate, where $\alpha$ and $\beta$ are arbitrary scalar fields,

Laplacian:

$$
\nabla^{2} \alpha=\frac{1}{a^{2}}\left(\frac{1}{\cos ^{2} \varphi} \frac{\partial^{2} \alpha}{\partial \lambda^{2}}+\frac{\partial^{2} \alpha}{\partial \varphi^{2}}-\tan \varphi \frac{\partial \alpha}{\partial \varphi}\right),
$$

Jacobian :

$$
J(\alpha, \beta)=\frac{1}{a^{2} \cos ^{2} \varphi}\left(\frac{\partial \alpha}{\partial \lambda} \frac{\partial \beta}{\partial \varphi}-\frac{\partial \alpha}{\partial \varphi} \frac{\partial \beta}{\partial \lambda}\right),
$$

Flux divergence:

$$
\begin{aligned}
\nabla \cdot(\alpha \nabla \beta)= & \frac{1}{a^{2} \cos ^{2} \varphi} \frac{\partial}{\partial \lambda}\left(\alpha \frac{\partial \beta}{\partial \lambda}\right) \\
& +\frac{1}{a^{2} \cos \varphi} \frac{\partial}{\partial \varphi}\left(\alpha \cos \varphi \frac{\partial \beta}{\partial \varphi}\right),
\end{aligned}
$$

we can rewrite equations (1)-(5) as follows after some differentiations and modifications.

$$
\begin{gathered}
\frac{\partial \eta}{\partial t}-J(\eta, \psi)+\nabla \cdot(\eta \nabla \chi)+\nabla \cdot\left(\dot{\sigma} \nabla \frac{\partial \psi}{\partial \sigma}\right) \\
+J\left(\dot{\sigma}, \frac{\partial \chi}{\partial \sigma}\right)+J(\sigma \alpha, \pi)=0
\end{gathered}
$$

$$
\begin{aligned}
\frac{\partial D}{\partial t} & -J(\eta, \chi)-\nabla \cdot(\eta \nabla \psi)+\nabla \cdot\left(\dot{\sigma} \nabla \frac{\partial \chi}{\partial \sigma}\right) \\
& -J\left(\dot{\sigma}, \frac{\partial \psi}{\partial \sigma}\right)+\nabla^{2}(K+\phi)+\nabla \cdot(\sigma \alpha \nabla \pi)=0,
\end{aligned}
$$$$
\frac{\partial \pi}{\partial t}-J(\pi, \psi)+\nabla \cdot(\pi \nabla \chi)+\frac{\partial \pi \dot{\sigma}}{\partial \sigma}=0,
$$$$
\frac{\partial T}{\partial t}-J(T, \psi)+\nabla \cdot(T \nabla \chi)-T \nabla^{2} \chi+p^{\kappa} \dot{\sigma} \frac{\partial \theta}{\partial \sigma}
$$$$
-\frac{\sigma \alpha}{C_{p}}\left\{\frac{\partial \pi}{\partial t}-J(\pi, \psi)+\nabla \cdot(\pi \nabla \chi)-\pi \nabla^{2} \chi\right\}
$$$$
-\frac{\dot{Q}}{C_{p}}=0,
$$

$$
\frac{\partial q}{\partial t}-J(q, \phi)+\nabla \cdot(q \nabla \chi)-q \nabla^{2} \chi+\dot{\sigma} \frac{\partial q}{\partial \sigma}-E=0,
$$

where $\eta=\zeta+f, \zeta=\nabla^{2} \psi, D=\nabla^{2} \chi$ and 


$$
\begin{aligned}
K= & \frac{1}{2}\left(u^{2}+v^{2}\right) \\
= & \frac{1}{2}\left[\nabla \cdot(\psi \nabla \psi)-\psi \nabla^{2} \psi+\nabla \cdot(\chi \nabla \chi)-\chi \nabla^{2} \chi\right. \\
& +J(\psi, \chi)] .
\end{aligned}
$$

As may be seen in equations (12)-(17), all the terms except the vertical advection terms are represented by Jacobian, Laplacian and flux divergence terms.

\section{The shallow water equation and its finite difference expressions for an icosahedral-hexagonal grid}

The simplest primitive equations model is the shallow water equations. To test the performance of our scheme in the primitive case, we have made a time integration of shallow water equation. The shallow water equations corresponding to the generalized equations (12)-(16) can be written as follows.

$$
\begin{aligned}
& \frac{\partial \eta}{\partial t}-J(\eta, \phi)+\nabla \cdot(\eta \nabla \chi)=0, \\
& \frac{\partial D}{\partial t}-J(\eta, \chi)-\nabla \cdot(\eta \nabla \psi)+V^{2}(K+\phi)=0, \\
& \frac{\partial \phi}{\partial t}-J(\phi, \phi)+\nabla \cdot(\phi \nabla \chi)=0 .
\end{aligned}
$$

To clarify the conservational properties of these equations, let us define the absolute potential vorticity, $Q$, the energy, $E$, and the absolute potential enstrophy, $Z$, as follows.

$$
\begin{aligned}
Q & =\frac{\eta}{\phi}, \\
E & =\frac{1}{2} \phi^{2}+\phi K, \\
Z & =\frac{1}{2} \phi Q^{2} .
\end{aligned}
$$

By multiplying (18) by $\psi$, (19) by $\chi$ and (20) by $\phi$, we get the equation referring to the energy,

$$
\frac{\partial E}{\partial t}-J(E, \psi)+\nabla \cdot(E \nabla \chi)=0 .
$$

And multiplying (18) by $\eta$ and rearrange it using (20), the equation for the absolute

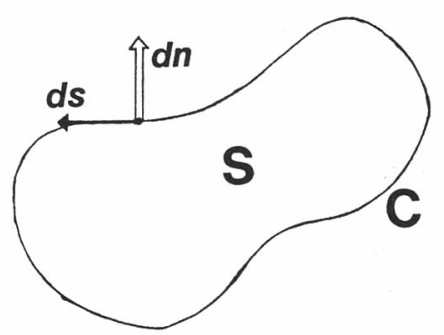

Fig. 2 Area integral and line integral.

potential enstrophy is obtained.

$$
\frac{\partial Z}{\partial t}-J(Z, \psi)+\nabla \cdot(Z \nabla \chi)=0
$$

According to the idea in the studies of Sadourny et al. (1968) and Williamson (1968), which will be reviewed briefly, Jacobian in differential form has the property,

$$
\iint_{S} J(\alpha, \beta) d S=\oint_{C} \alpha \frac{\partial \beta}{\partial s} d s .
$$

Here, as shown in Fig. 2, $S$ is an arbitrary domain bounded by curve $C$, and $\partial / \partial s$ is referred to the derivative along curve $C$. At the same time, the flux divergence and Laplacian terms can be transformed using Gauss' theorem as follows.

$$
\begin{aligned}
& \iint_{S} \nabla \cdot(\alpha \nabla \beta) d S=\oint_{C} \alpha \frac{\partial \beta}{\partial n} d s, \\
& \iint_{S} \nabla^{2} \alpha d S=\iint_{S} \nabla \cdot(\nabla \alpha) d S=\oint_{C} \frac{\partial \alpha}{\partial n} d s,
\end{aligned}
$$

where $\partial / \partial n$ means the derivative normal to curve $C$. It is easily seen that if the area integral is done over the entire surface of the sphere, $\Sigma$, all the integrals (26), (27) and (28) vanish.

$$
\begin{aligned}
& \iint_{\Sigma} J(\alpha, \beta) d S=0, \\
& \iint_{\Sigma} \nabla \cdot(\alpha \nabla \beta) d S=0, \\
& \iint_{\Sigma} \nabla^{2} \alpha d S=0 .
\end{aligned}
$$

Applying these properties to equations (20), (18), (19), (24) and (25), total mass, total absolute vorticity, total divergence, total energy and total absolute potential enstrophy are conserved over the entire surface of the 


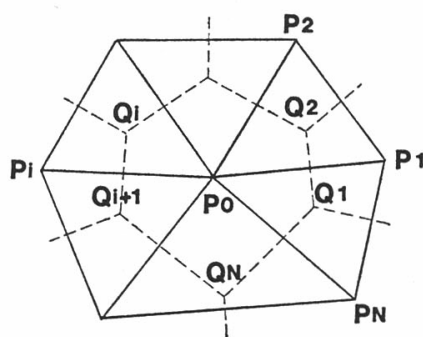

Fig. 3 Configuration of a hexagonal grid element.

earth. It is better to formulate the finite difference so as to satisfy these conservation properties mentioned above as far as possible. Especially, as Sadourny (1975) emphasized, potential enstrophy conservation is the most important.

Fig. 3 shows the configuration of a hexagonal (pentagonal at the vertices of the icosahedron) grid element. $Q_{i}$ is the equidistant point from $P_{0}, P_{i-1}$ and $P_{i}$ such that $\overline{Q_{i} Q_{i+1}}$ is the perpendicular bisector of the arc $\bar{P}_{0} P_{i}$. All the variables are defined at the points $P_{i}(i=0,1,2, \cdots, N ; N=5$ or 6$)$. If we represent the finite difference expression of Jacobian, $J(\alpha, \beta)$ by $\boldsymbol{J}(a, b)$, we get from equation (26),

$$
\boldsymbol{J}(a, b)=\frac{1}{s_{*}} \sum_{i=1}^{N} \frac{A_{0}+A_{i}}{2}\left(b_{i+1}-b_{i}\right),
$$

where ' $A$ ' is the value corresponding to the variable a at the ' $P$ ' point, $s_{*}$ the area of the inner small polygon surrounded by $Q_{1}, Q_{2}, \cdots, Q_{N}$. The value $b_{i}$ at a point $Q_{i}$ is defined by using the values ' $B$ ' at the ' $P$ ' points as follows,

$$
b_{i}=\left(B_{0}+B_{i}+B_{i-1}\right) / 3 \text {. }
$$

By substituting (33) into (32), we can obtain the finite difference formulation of Jacobian,

$$
\boldsymbol{J}(a, b)=\frac{1}{6 s_{*}} \sum_{i=1}^{N}\left(A_{0}+A_{i}\right)\left(B_{i+1}-B_{i-1}\right) .
$$

This expression is easily transformed into

$$
\boldsymbol{J}(a, b)=\frac{1}{6 s_{*}} \sum_{i=1}^{N} A_{i}\left(B_{i+1}-B_{i-1}\right),
$$

or

$$
\boldsymbol{J}(a, b)=\frac{1}{6 s_{*}} \sum_{i=1}^{N} B_{i}\left(A_{i-1}-A_{i+1}\right) .
$$

The expression (34) satisfies the conservation properties for the total vorticity, total diver- gence and total mass, the expression (35) for the total absolute enstrophy and (36) for the total energy.

The finite difference scheme for the flux divergence is derived from the equation (27).

$$
\boldsymbol{F}(a, b)=\frac{1}{s_{*}} \sum_{i=1}^{N} \frac{l_{i}}{L_{i}} \frac{A_{0}+A_{i}}{2}\left(B_{i}-B_{0}\right) .
$$

Where $L_{i}$ is the length of the $\operatorname{arc} \overline{P_{0} P_{i}}$ and $l_{i}$ is that of $\overline{Q_{i} Q_{i+1}} \cdot \boldsymbol{F}(a, b)$ is suitable to conserve the total vorticity, total divergence and total mass.

The finite difference approximation for Laplacian is obtained from the equation (31). That is,

$$
\nabla^{2} \alpha=\frac{1}{s_{*}} \sum_{i=1}^{N} \frac{l_{i}}{L_{i}}\left(A_{i}-A_{0}\right) .
$$

This expression also satisfies conservation property shown by equation (31). After all we may say that the scheme used in this paper conserves exactly the total absolute vorticity, total divergence and total mass, and almost exactly the total energy and total absolute enstrophy.

These finite difference approximations, that is, (35) for Jacobian, (37) for flux divergence and (38) for Laplacian, are employed in the integration of the equations (18)-(20) and kinetic energy expression (17). A line integral is done along the inner small polygon surrounded by $Q_{1}, Q_{2}, \cdots, Q_{N}$. This treatment is different from that of Sadourny et al. (1968) and Williamson (1968), because they made a line integral along the outer large polygon.

\section{Results of numerical experiments}

With the use of the finite difference scheme presented in the previous section, we have made some numerical experiments for the divergent barotropic (shallow water) case. The initial condition is the so-called RossbyHaurwitz wave, which has been used by Phillips (1959) and subsequently by many investigators. There is no divergence in the initial state.

$$
\begin{aligned}
\psi= & A \sin (m \lambda) P_{n}^{m}(\sin \varphi) \\
& -\frac{n(n+1) C+2 \omega}{n(n+1)-2} a^{2} \sin \varphi,
\end{aligned}
$$




$$
\chi=0,
$$

where $A$ is an arbitrary constant and set to be $1000 \mathrm{~m}^{2} \cdot \mathrm{sec}^{-1}, m$ is wave number, $n=m+1$, $a$ and $\omega$ are the radius and angular velocity of the earth respectively, $C$ phase velocity of the wave which is set to be 0 in this experiment. This wave, in a non-divergent barotropic atmosphere, propagates with a constant east-west phase velocity, $C$, without any change of wave shape. When this wave pattern is applied in a divergent case, however, it is difficult to get the precise analytical solution.

To get the solution of equation (18) and (19), we must solve the Poisson's equation in the icosahedral-hexagonal grid. In the earlier stage of this study, the solution was obtained by a sequential relaxation. From the preliminary numerical test, however, the relaxation method produced systematic large errors. So, finally, the inverse matrix method is applied in order to obtain the solution.

Each side of the spherical icosahedron, which is a great circle arc of about 63 degrees, is divided into 10 in this study. The grid distances are about 6.3 degrees $(690 \mathrm{~km})$ and the time step is set to be 10 minutes. The main part of time integration is done by the leap-frog scheme. At every 144 time steps ( 1 day forecast), the Euler-backward scheme is inserted for 6 steps ( 1 hour). The time integration is extended up to 100 days starting from the Rossby-Haurwitz wave of a certain wave number. All the calculations are processed under the double precision computer memories.

The results for the initial wave number 2 case are shown in Figs. 4 and 5. In Fig. 4 we can see a wave number 5 disturbance near the equator and some irregularities even at the initial state, but these are because of a crude interpolation from the icosahedralhexagonal grid to the latitude-longitude grid for drawing the figures and not because of the forecast model. Starting from a nondivergent initial state, as can be seen in Fig. 5 , there appears a weak divergence and it reaches a balanced state after several days without more intensification. The wave pattern propagates slowly to the east with the phase speed of about 3-4 degrees per day.

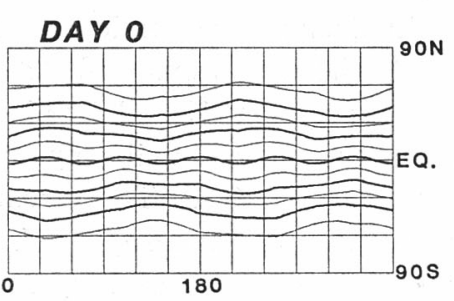

DAY36

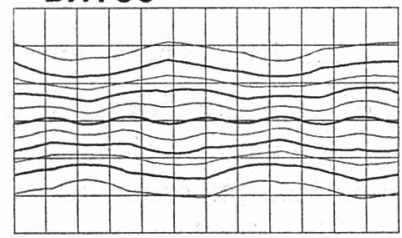

DAY72

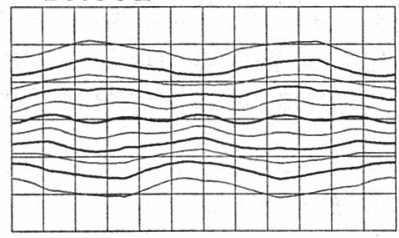

DAY 12

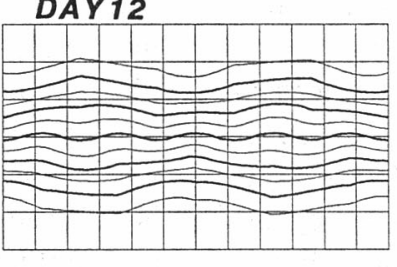

DAY 48

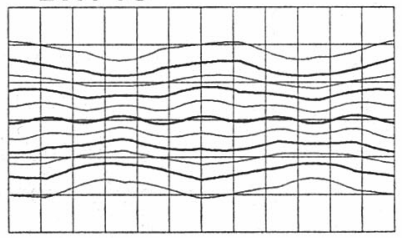

DAY 84

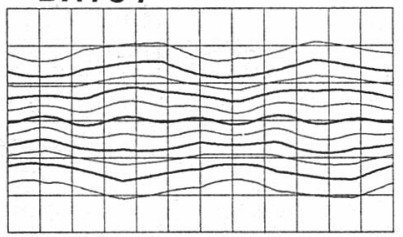

DAY24

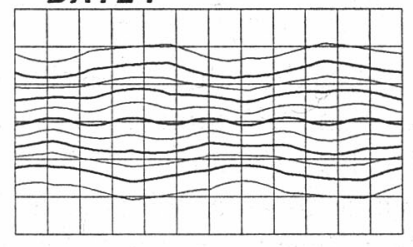

DAY60

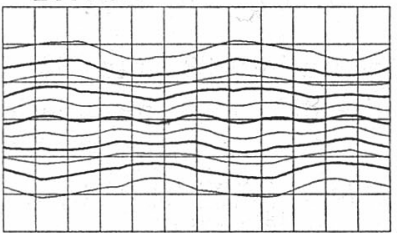

DAY96

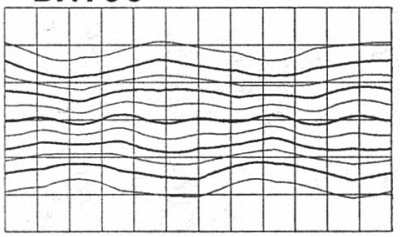

Fig. 4 Time change of stream function field for wave number 2. Abscissa is the longitude and ordinate is the latitude. Contour interval: $1 \times 10^{8} \mathrm{~m}^{2} \cdot \mathrm{sec}^{-1}$. 

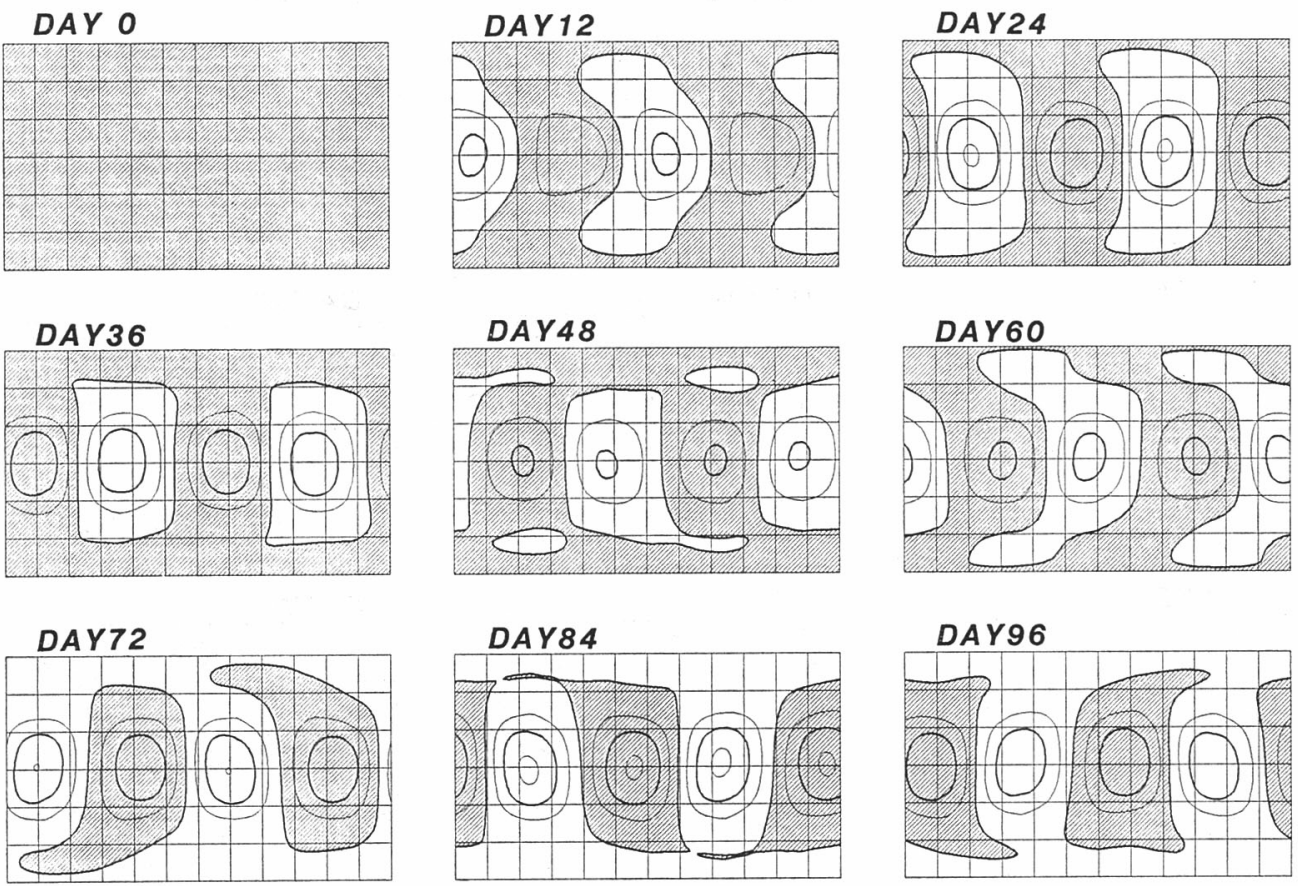

Fig. 5 Same as Fig. 4 but of the velocity potential field. Contour interval: $1 \times 10^{6} \mathrm{~m}^{2} \cdot \mathrm{sec}^{-1}$. Negative regions are shaded.

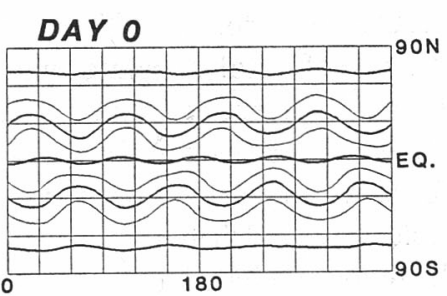

DAY 12

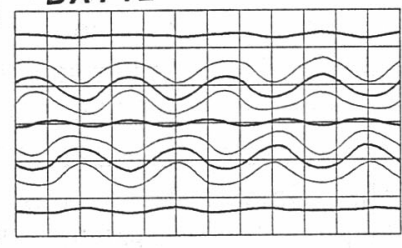

DAY 36
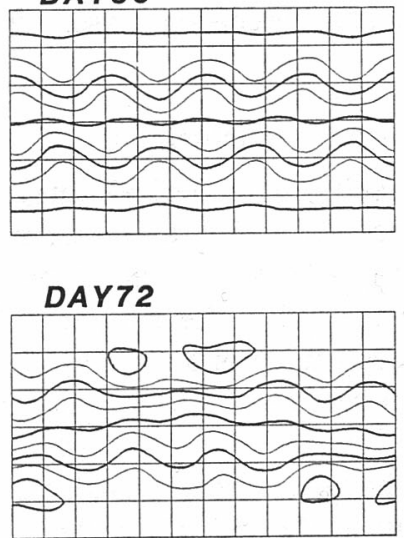

DAY48
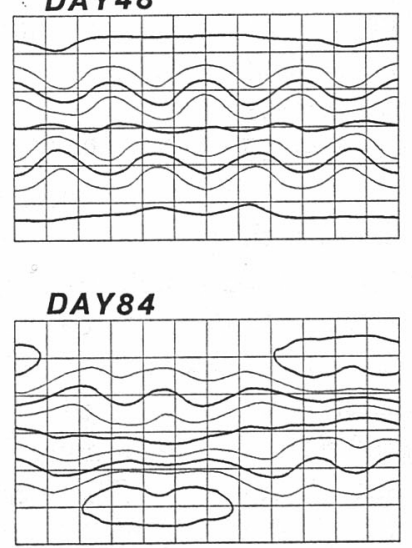

DAY24
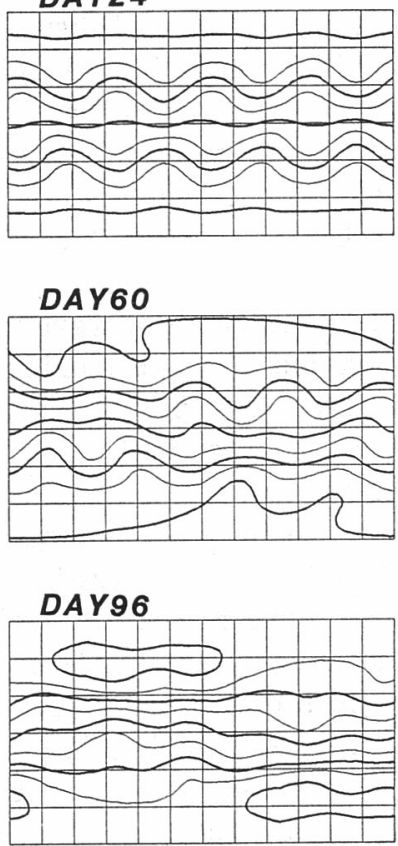

Fig. 6 Same as Fig. 4 but for the wave number 4 . Contour interval: $5 \times 10^{7} \mathrm{~m}^{2} \cdot \mathrm{sec}^{-1}$. 

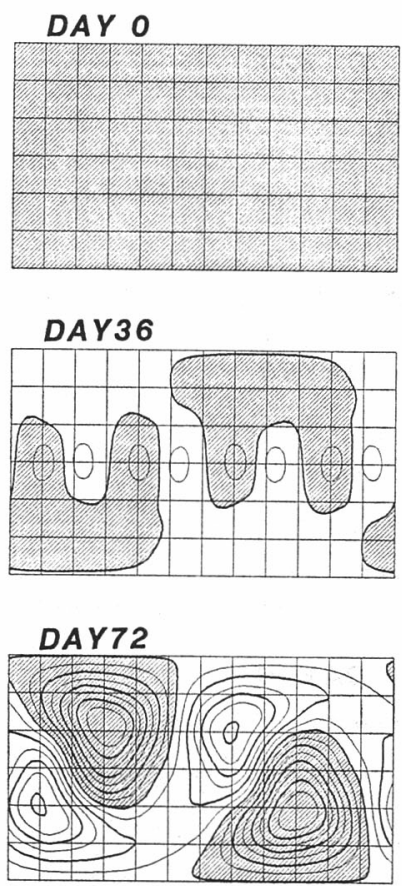

DAY 12

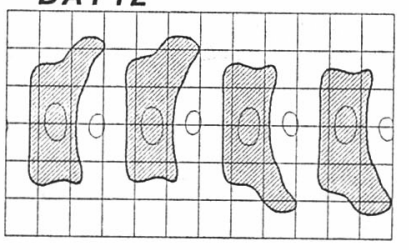

$D A Y 48$

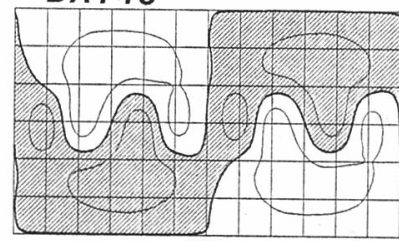

DAY 84

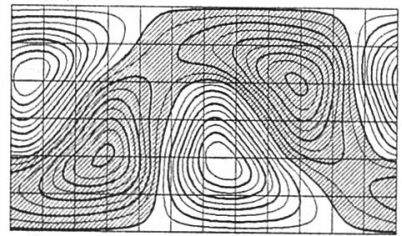

DAY24

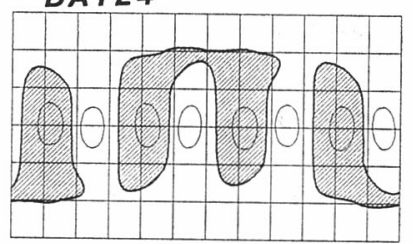

DAY60

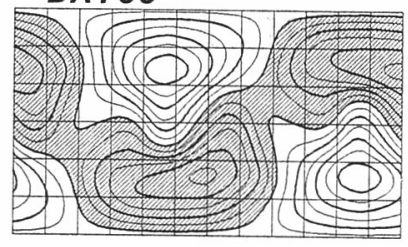

DAY96

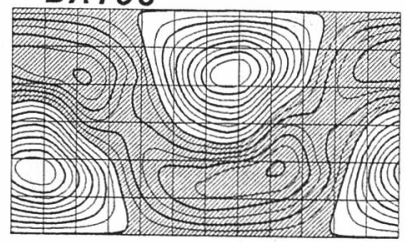

Fig. 7 Same as Fig. 5 but for the wave number 4 . Contour interval : $2 \times 10^{5} \mathrm{~m}^{2} \cdot \mathrm{sec}^{-1}$.

The wave pattern is well maintained after 100 days integration.

Figs. 6 and 7 show the results for the case in which wave number 4 disturbance is given at the initial time. Initially given pattern is maintained for about 48 days of integration moving slowly to the west with the phase speed of 1 degree per day. After that time a disturbance of wave number 1 is intensified in the middle latitudes and finally conceals the disturbance of wave number 4 . The intensification of wave number 1 is evident in the divergent part. This wave moves quickly to the west with a phase speed of 25 degrees per day.

As shown by Hoskins and Hollingsworth (1973), the larger scale Rossby-Haurwitz wave (less than wave number 3 , but changed with the parameters) are stable in the barotropic case without dissipation, but the smaller scale waves become unstable. The predicted results for the waves of wave number 2 and 4 represent to be reasonable, even though the resolution of our model is quite coarse.

The properties of material conservation are shown in Fig. 8. The total mass is con- served satisfactorily. The total energy is maintained within less than 0.001\% error throughout 100 days forecats. The error in the conservation of total absolute enstrophy is not so good as total energy, but it remains within $0.01 \%$ error for the case of wave number 2 (stable case) and $0.1 \%$ for wave number 4 (unstable case). With the use of single precision, the errors included in the conservation properties are $0.1-0.6 \%$, which are much larger than double precision cases showed here.

\section{Discussions and conclusions}

From the results of numerical integration described in the previous section, we may be able to draw the conclusion that the finite difference scheme with the icosahedral-hexagonal grid gives the satisfactory approximation to the integration of the shallow water equations.

There are, however, some problems to be solved in the unstable (initial wave number greater than 4) cases. As it can be seen in Fig. 7, initially given wave number 4 disturbance breaks down into wave number 1 . 


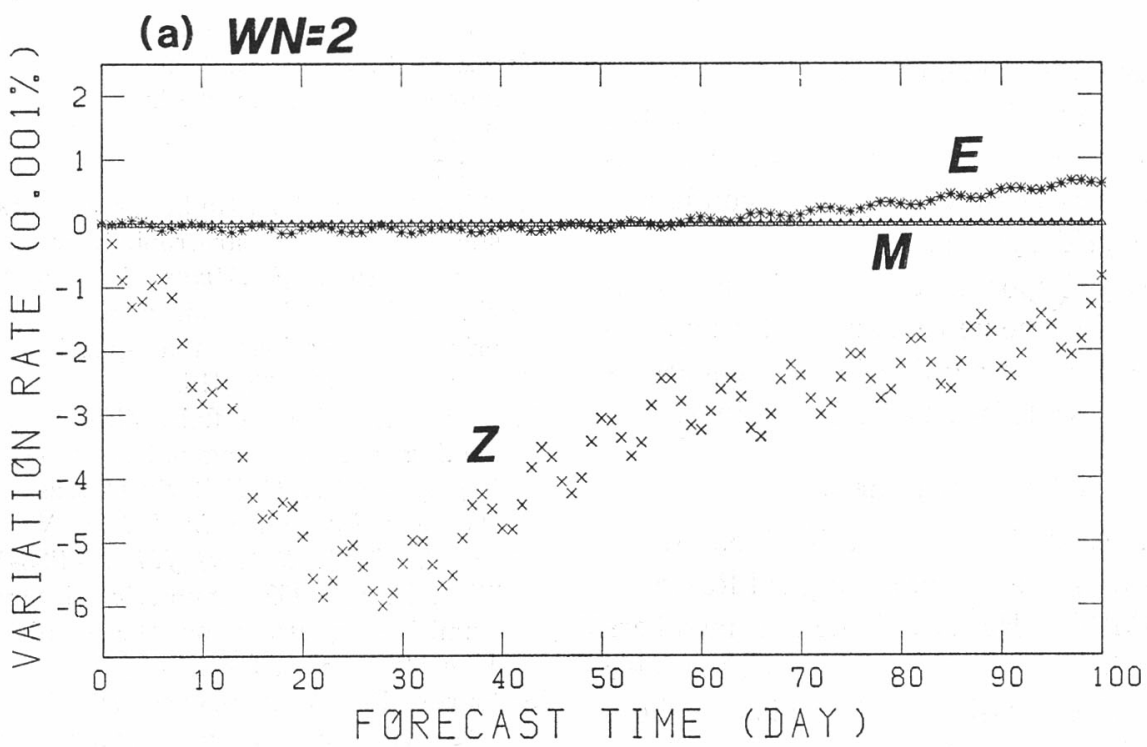

(b) $W N=4$

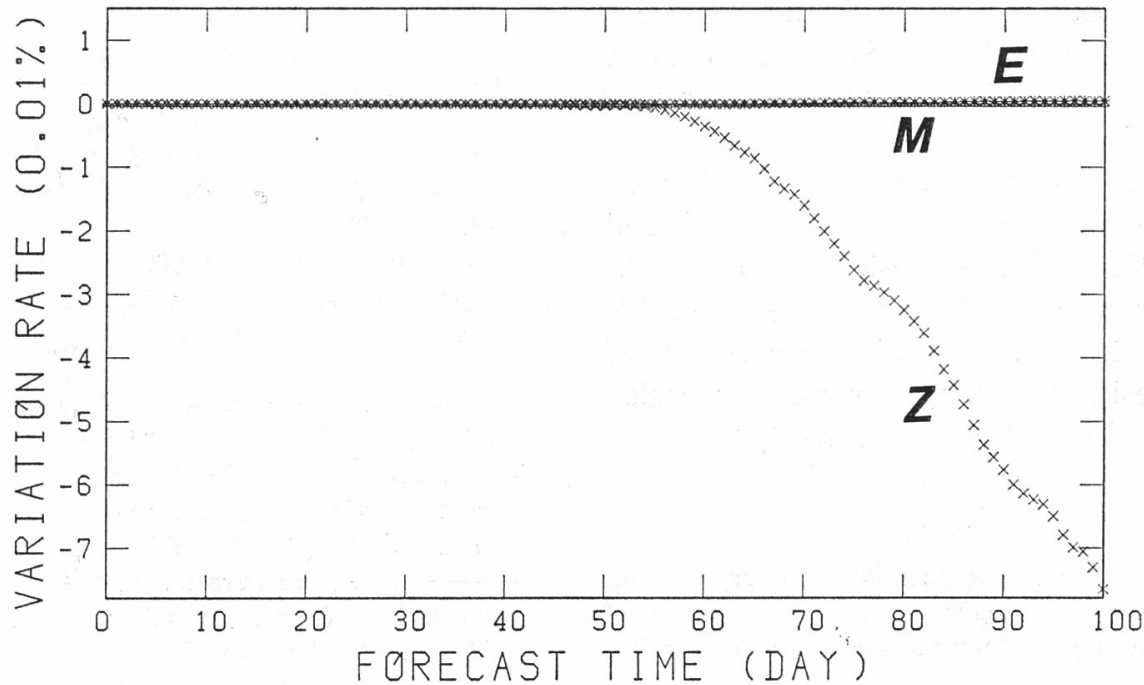

Fig. 8 Time change of total mass $(M)$, total energy $(E)$ and total potential enstrophy $(Z)$ for wave number 2 (a) and 4 (b). Notice that the ordinate is scaled by each $0.001 \%$ in (a) and $0.01 \%$ in (b).

The phase relation of this wave between northern and southern hemisphere is out of phase. In the low latitude region, these waves from both hemispheres intersect each other to form a wave number 2 disturbance. We have made some experiments for different initial wave numbers. In the case of wave number 6 , the disturbance of wave number 1 prevails after long integration. If we set the wave number of initial disturbance to be 7 , wave number 2 disturbance takes place of initially given wave. In the case of wave number 5, which is expected to be unstable by the theory, the wave is stable rather than unstable. As easily to be seen, the icosahedralhexagonal grid system has the symmetry of wave number 5 in higher latitudes and wave number 10 in lower latitudes. The phase relation of grid symmetry between both hemisperes is out of phase. From these facts, we 
may guess that the errors in the computational accuracy stemmed from the interaction between configuration of the grid system and the unstable waves. It is important to diminish these errors coming from the configuration of the grid system.

The future importance of this icosahedralhexagonal grid scheme remains to its application to the more general primitive equations for the large scale atmospheric motions.

\section{Acknowledgements}

This investigation started in 1983 when Y. Masuda was in the Meteorological Research Institute (MRI). $\mathrm{He}$ is indebted to members of Forecast Research Division of MRI for their many helpful discussions. And also authors express their gratitude to members of Typhoon Research Division of MRI for their useful comments about the results of numerical experiments. The authors would like to thank the referees for their constructive advices in revising this paper.

Computations are made with the HITAC M200-H, M280-D and S810/10 of MRI and FACOM M180 in System Design Company Ltd.. Y. Masuda, one of the author, express his thanks to Mr. M. Okazaki, the president of System Design Company, for his permission to use the FACOM computer freely.

\section{References}

Arakawa, A., 1966: Computational design for longterm numerical integration of the equations of fluid motion: Two-dimensional incompressible flow, Part I. J. Compu. Phys., 1, 119-143.

Gates, W. L. and C.A. Riegel, 1962: A study of numerical errors in the integration of barotropic flows on a spherical grid. J. Geophys. Res., 67, 773-784.

Hoskins, B. J. and A. Hollingsworth, 1973: On the simple example of barotropic instability of Rossby wave motion. J. Atmos. Sci., 30, 150-153.

Kurihara, Y., 1965: Numerical integration of the primitive equations on a spherical grid. Mon. Wea. Rev., 93, 399-415.

Masuda, Y., 1969: A finite difference scheme by making use of hexagonal mesh-points. Proceedings of the WMO/IUGG Symposium on Numerical Weather Prediction in Tokyo, Nov. 26-Dec. 4, 1968. Tech. Rep. of JMA, VII35-VII44.

Phillips, N. A., 1959: Numerical integration of the primitive equations on the hemisphere. Mon. Wea. Rev., 87, 333-345.

Sadourny, R., A. Arakawa and Y. Mintz, 1968 : Integration of the nondivergent barotropic vorticity equation with an icosahedral-hexagonal grid for the sphere. Mon. Wea. Rev., 96, 351356.

, 1969: Numerical integration of the primitive equations on a spherical grid with hexagonal cells. Proceedings of the WMO/IUGG Symposium on Numerical Weather Prediction in Tokyo, Nov. 26-Dec. 4, 1968. Tech. Rep. of JMA, VII45-VII52.

and P. Morel, 1969: A finite-difference approximation of the primitive equations for a hexagonal grid on a plane. Mon. Wea. Rev., 97, 439-445.

- 1975: Compressible model flows on the sphere. J. Atmos. Sci., 32, 2103-2110.

Williamson, D.L., 1968: Integration of the barotropic vorticity equation on a spherical geodesic grid. Tellus, 10, 642-653.

, 1969: Integration of the primitive barotropic model over a spherical geodesic grid. Mon. Wea. Rev., 98, 512-520. 